\title{
Determining Whether a Simplicial 3-Complex Collapses to a 1-Complex Is NP-Complete
}

\author{
Rémy Malgouyres ${ }^{1}$ and Angel R. Francés ${ }^{2}$ \\ ${ }^{1}$ Univ. Clermont 1, Laboratoire d'Algorithmique et Image (LAIC, EA2146), \\ IUT dpartement informatique Campus des Cézeaux, \\ B.P. 86, 63172 Aubière cedex, France \\ remy.malgouyres@laic.u-clermont1.fr \\ http://laic.u-clermont1.fr/ ${ }^{\mathrm{mr} /}$ \\ ${ }^{2}$ Dpto. Informática e Ingeniería de Sistemas, Facultad de Ciencias, Universidad de \\ Zaragoza. C/. Pedro Cerbuna, 12. E-50009 Zaragoza, Spain \\ afrances@posta.unizar.es
}

\begin{abstract}
We show that determining whether or not a simplicial 2complex collapses to a point is deterministic polynomial time decidable. We do this by solving the problem of constructively deciding whether a simplicial 2-complex collapses to a 1-complex. We show that this proof cannot be extended to the 3D case, by proving that deciding whether a simplicial 3 -complex collapses to a 1 -complex is an $N P$-complete problem.
\end{abstract}

Keywords: Simplicial Topology, Collapsing, Computational Complexity, $N P$-completeness.

\section{Introduction}

In the framework of digital topology, several authors have considered discrete deformation retraction and collapsing, in particular for characterizing simple points (K97, KR01, B99]). All of these authors emphasize the importance of finding efficient algorithms for deciding whether an object can be shrunk on another object (see also [F00]). In this paper, we investigate the case when "object" is a simplicial complex, and "shrunk" means collapsed.

In [EG96], a generalized collapsing problem is proved $N P$-complete for simplicial 2-complexes (see Theorem 1 below). However, this problem is rather artificial and does not, contrary to the collapsing problems considered here, arise from topologists' questions on topology preservation.

This paper is intended to be readable both by topologists and by specialists in computational complexity. For this purpose, we find two (relatively long) sections recalling basic notions concerning collapsing of simplicial complexes and $N P$-completeness.

Then we investigate the $2 \mathrm{D}$ case, showing that deciding whether or not a simplicial 2-complex collapses to a 1-complex is polynomial. It follows that deciding whether a 2 -complex collapses to a point is also polynomial. 
Finally, we prove that the corresponding 3D problem of deciding whether or not a simplicial 3-complex collapses to a 1 -complex is $N P$-complete.

\section{Basic Notions of Simplicial Topology}

\subsection{Simplicial Complexes}

A (finite abstract) simplicial complex $\mathcal{C}$ is a couple $\mathcal{C}=(V, S)$, where $V=$ $\left\{v_{1}, \ldots, v_{p}\right\}$ is a finite set, and $S$ is a set of subsets of $V$, containing all singletons, and such that any subset of an element of $S$ is also an element of $S$. An element of $V$ is called a vertex of $\mathcal{C}$, and an element of $S$ is called a cell of $\mathcal{C}$ or a simplex of $\mathcal{C}$. A cell $s$ of $\mathcal{C}$ with cardinality $d+1$ is called a $d$-cell of $\mathcal{C}$, and the number $d$ is called the dimension of $s$. The dimension of $\mathcal{C}$ is the maximal dimension of its cells. For $k \in \mathbb{N}$, we call a simplicial $k$-complex any simplicial complex with dimension less than or equal to $k$. In the sequel, all considered simplicial complexes are finite.

Let $\mathcal{C}=(V, S)$ be a simplicial complex. If $s^{\prime} \subset s$, with $s \in S$, we say that $s^{\prime}$ is a face of $s$. If in addition we have $s \neq s^{\prime}$, then $s^{\prime}$ is called a proper face of $s$. Finally, if $s^{\prime}$ is a proper face of $s, s^{\prime}$ is a $(d-1)$-cell, and $s$ is a $d$-cell, we call $s^{\prime}$ a maximal proper face of $s$.

\subsection{Collapsing}

Let $\mathcal{C}=(V, S)$ be a simplicial complex and let $s^{\prime}$ be a maximal proper face of a cell $s$ in $\mathcal{C}$. We say that $s^{\prime}$ is a free face of $s$ in $\mathcal{C}$ (or merely a free face for short) if $s^{\prime}$ is a proper face of no cell in $\mathcal{C}$ except $s$.

If $s^{\prime}$ is a free face of $s$ in $\mathcal{C}$, we can define a new simplicial complex $\mathcal{C}^{\prime}=\left(V^{\prime}, S^{\prime}\right)$, called an elementary collapse of $\mathcal{C}$, by considering the set $V^{\prime}$ of vertices of $V$ which belong to some cell of $\mathcal{C}$ which is different from $s$ and $s^{\prime}$, and the set $S^{\prime}=S-\left\{s, s^{\prime}\right\}$. We say also that there is an elementary collapse of $\mathcal{C}$ on $\mathcal{C}^{\prime}$, and that the collapse is across $s$ from $s^{\prime}$. Though we do not need these facts, we mention that it is known (and not difficult) that if $\mathcal{C}^{\prime}$ is an elementary collapse of $\mathcal{C}$, then $\mathcal{C}^{\prime}$ is a strong deformation retract of $\mathcal{C}$, so that $\mathcal{C}$ and $\mathcal{C}^{\prime}$ have the same homotopy type.

Now, given $\mathcal{C}=(V, S)$ a simplicial complex and $\mathcal{C}^{\prime}=\left(V^{\prime}, S^{\prime}\right)$ a subcomplex of $\mathcal{C}$ (i.e. $V^{\prime} \subset V$ and $S^{\prime} \subset S$ ), we say that $\mathcal{C}$ collapses on $\mathcal{C}^{\prime}$ if there is a finite sequence $\left(\mathcal{C}_{0}, \ldots, \mathcal{C}_{n}\right)$ of simplicial subcomplexes of $\mathcal{C}$, such that $\mathcal{C}=\mathcal{C}_{0}, \mathcal{C}^{\prime}=\mathcal{C}_{n}$, and for $i=1, \ldots, n$ the complex $\mathcal{C}_{i}$ is an elementary collapse of the complex $\mathcal{C}_{i-1}$. If in addition the complex $\mathcal{C}^{\prime}$ is 1 -dimensional, we say that $\mathcal{C}$ collapses to a 1 -complex. If $\mathcal{C}^{\prime}$ is reduced to a single vertex $\left(\mathcal{C}^{\prime}=(\{v\},\{\{v\}\})\right)$, we say that $\mathcal{C}$ collapses to a point or, simply, that $\mathcal{C}$ is collapsible.

Next we give some examples of both collapsible and non-collapsible complexes that will be used in Section 3. All of these complexes are variations around the well-known Bing's house introduced in B64. 
Example 1. Let us consider the object depicted in Figure 1(a), called here the Bing's house with two thin walls. This is a $2 D$ object, which can be described as follows. The object is a "building" with two rooms, the upper room and the lower room. We can enter in the upper room by a tunnel through the lower room. Similarly, we can enter in the lower room by a tunnel through the upper room. Finally, two thin walls (i.e. 2D walls) are added between the tunnel through each room and an exterior wall of the building, so that both rooms are simply connected. In order to realize the Bing's house with a simplicial complex $\mathcal{B}=$ $(V, S)$, we can break all the rectangular pieces of walls into triangles (i.e. we can triangulate the $2 D$ walls). Then we take for $V$ the set of all vertices of the obtained triangles, and as simplexes (elements of $S$ ), the singletons of $V$ as 0-cells, pairs of extremities of edges as 1-cells, and the sets consisting of the three vertices of triangles as 2 -cells. The obtained simplicial 2 -complex $\mathcal{B}$ is not collapsible since no cell of $\mathcal{B}$ has a free face, but it has the homotopy type of a point. For this, first thicken all its walls (this is the inverse operation of a collapse) and observe that the resulting $3 D$ object is a 3-ball, which collapses to a point. The next example shows that it is sufficient to thicken only one appropriate wall of $\mathcal{B}$ to check this result.

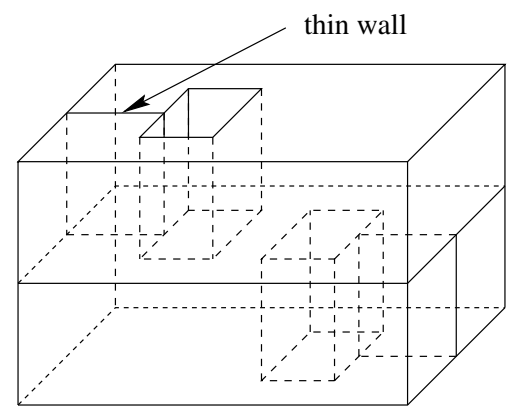

(a) The Bing's house with two thin walls.

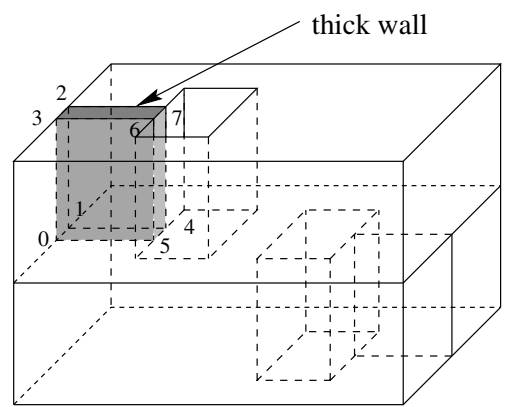

(b) The Bing's house with one thick wall and one thin wall.

Fig. 1. Examples of simplicial complexes

Example 2. Now let us consider the object depicted in Figure 1(b), It is called the Bing's house with one thick wall. It is similar to the Bing's house with two thin walls, except that one of the walls is thickened to get a 3D paralelepipedic wall $W$, as represented in Figure 1(b). In order to get a simplicial complex to represent the Bing's house with one thick wall, we proceed as in Example 1 for the $2 D$ parts, but for the $3 D$ wall, we consider a particular triangulation $\mathcal{W}$ consisting of twelve 3-cells, which is as follows. Consider the vertices numbers of the cube representing the thick wall of Figure 1(b). This cube is also represented in Figure 2(a), where it has been subdivided into four triangular prisms by adding a new edge $\{8,9\}$ with extremities at the barycentres of the rectangles $(0,3,5,6)$ and $(1,2,4,7)$, respectively, and joining these barycentres to the vertices of the corresponding rectangles. Then, each of these prisms is in turn subdivided into three 


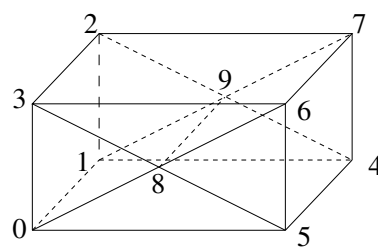

(a)

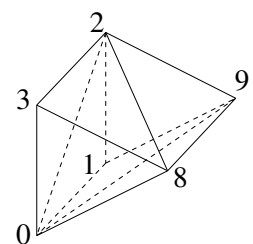

(b)

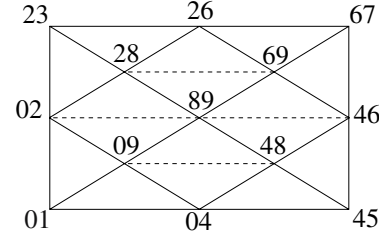

(c)

Fig. 2. Decomposition into simplexes of a cube

3-cells as follows. First, divide each rectangular face of the prism with a diagonal containing the vertex with the lowest label, and then consider all tetrahedra thus obtained. For example, the prism with vertices $0,1,2,3,8,9$ is decomposed into the 3 -cells $\{0,2,3,8\},\{0,2,8,9\}$ and $\{0,1,2,9\}$ (see Figure 2(b)). Thus the Bing's house with one thick wall is realized as a simplicial 3-complex $\mathcal{B}_{2}$ which is collapsible.

Indeed, we can first remove each prism from the free $2 D$ faces on the rectangles $(0,1,2,3),(2,3,6,7),(4,5,6,7)$ and $(0,1,4,5)$. For example, the prism with vertices $0,1,2,3,8,9$ can be removed by a sequence of elementary collapses across the following cells: $\{0,1,2,9\}$ from $\{0,1,2\}$, then $\{0,2,3,8\}$ from $\{0,2,3\}$, afterwards $\{0,2,8,9\}$ from $\{0,2,8\}$, and finally $\{0,2,9\}$ from $\{0,2\}$, and similarly for the other prisms. After this process, edges $\{2,3\},\{4,5\}$ and $\{6,7\}$ become free faces, from which we can remove rectangles $(2,3,8,9),(4,5,8,9)$ and $(6,7,8,9)$, respectively. Namely, for rectangle $(2,3,8,9)$ collapse the 2 -cell $\{2,3,8\}$ from $\{2,3\}$ and, afterwards, $\{2,8,9\}$ from $\{2,8\}$. Now we can remove the rectangle $(0,1,8,9)$ from the recently created free face $\{8,9\}$ in a similar way. Note that the $2 D$ faces representing the rectangles $(0,3,5,6)$ and $(1,2,4,7)$, up to now, remain in the obtained 2-complex and have no free faces. This remaining 2-complex $\mathcal{B}_{2}^{\{0,1\}}$ has $\{0,1\}$ as a free face, which enables us to begin to remove the bottom part of the Bing's house, and subsequently, all the object until we get a single vertex.

Remark 1. Notice that despite the 2 -cells lying in the rectangles $(0,3,5,6)$ and $(1,2,4,7)$ are free faces of $\mathcal{B}_{2}$, none of them can be used in order to collapse the Bing's house with one thick wall to a point.

To check this, let us consider the intersection of the thick wall $W$ with a plane $P$ parallel to rectangles $(0,3,5,6)$ and $(1,2,4,7)$. If $\mathcal{W}=\left(V, S_{1} \cup S_{2}\right)$ is the triangulation of $W$ described in Example 2 , where $S_{1}$ is the set of cells of $\mathcal{W}$ lying in the rectangles $(0,3,5,6)$ and $(1,2,4,7)$ and $S_{2}$ contains the remaining cells of $\mathcal{W}$, the intersection of $P$ with the cells in $S_{2}$ induces a subdivision of $W \cap P$ that can be extended (without introducing new vertices) to get a collapsible simplicial 2-complex $\mathcal{R}$. This complex is shown in Figure 2(c), where the label of each vertex has been made up of the vertices numbers of the corresponding edge in $\mathcal{W}$, and the solid lines represent the intersection of a triangle in the set $S_{2}$ with the plane $P$. 
Now, let $\mathcal{B}_{2}=\mathcal{C}_{0}, \mathcal{C}_{1}, \ldots, \mathcal{C}_{p}$ be a sequence of subcomplexes of the Bing's house $\mathcal{B}_{2}$ such that $\mathcal{C}_{i-1}$ elementary collapses on $\mathcal{C}_{i}$, for $1 \leq i \leq p$, and $\mathcal{C}_{p}$ consists of a single point. Moreover, let $i_{0}$ be the lowest index such that the corresponding collapse is across a cell not contained in the thick wall $\mathcal{W}$. Notice that $i_{0}>1$ necessarily and $\mathcal{C}_{i_{0}-1}$ collapses on $\mathcal{C}_{i_{0}}$ from either the edge $\{0,1\}$ or from an edge in the border of the rectangle $(0,3,5,6)$ or $(1,2,4,7)$. Finally, for $0 \leq i<i_{0}$, let us consider the 2 -complex $\mathcal{R}_{i}$ obtained by intersecting the cells of $\mathcal{W}_{i}=\mathcal{W} \cap \mathcal{C}_{i}$ with the plane $P$ as above.

Then, for $0<i<i_{0}$, if $\mathcal{C}_{i-1}$ collapses on $\mathcal{C}_{i}$ (or, equivalently, $\mathcal{W}_{i-1}$ collapses on $\mathcal{W}_{i}$ ) across a cell $s$ from the free face $s^{\prime}$, it is not difficult to check that $s^{\prime} \in S_{2}$ if and only if $\mathcal{R}_{i}$ is also an elementary collapse of $\mathcal{R}_{i-1}$, and the collapse is across $s \cap P$ from $s^{\prime} \cap P$. For example, the elementary collapse of $\mathcal{B}_{2}$ across $\{0,2,3,8\}$ from $\{0,2,3\}$ corresponds with the collapse of $\mathcal{R}=\mathcal{R}_{0}$ across $\{02,23,28\}$ from $\{02,23\}$. Hence, any collapsing sequence of $\mathcal{R}$ to the point $\{01\}$ (i.e., to the complex $(\{01\},\{\{01\}\}))$ corresponds with a collapsing sequence of $\mathcal{B}_{2}$ on the complex $\mathcal{B}_{2}^{\{0,1\}}$, in which the edge $\{0,1\}$ is a free face, and conversely. On the other hand, if $\mathcal{C}_{i-1}$ collapses on $\mathcal{C}_{i}$ from a face $s^{\prime} \in S_{1}$, then $\mathcal{R}_{i}$ is obtained by removing from $\mathcal{R}_{i-1}$ the cell $s \cap P$ without deleting any of its faces. For example, the collapse across $\{0,2,3,8\}$ from $\{0,3,8\}$ corresponds with the deletion of the 2 -cell $\{02,23,28\}$ in $\mathcal{R}$. In this case $\mathcal{R}_{i}$ is not longer collapsible since it has a hole, and hence $\mathcal{C}_{i}$ cannot collapse on $\mathcal{B}_{2}^{\{0,1\}}$ just across elementary collapses from cells in the set $S_{2}$. Moreover, it can be proved, by induction on the number of collapses, that any elementary collapse of $\mathcal{C}_{i}$ from a face in $S_{1}$ will not destroy the hole in $\mathcal{R}_{i}$ (on the contrary, it will create a new hole or, at most, it will merge two holes) and, in addition, that the edges of the rectangles $(0,3,5,6)$ and $(1,2,4,7)$ never become free. This is a contradiction with our hypothesis, since then the edge $\{0,1\}$ cannot become free either.

This shows, in particular, that in order to collapse the Bing's house with one thick wall $\mathcal{B}_{2}$ to a point, the edge $\{0,1\}$ necessarily becomes a free face at some step of the collapsing sequence.

Example 3. We finish this section introducing a 3 -complex $\mathcal{B}_{3}$ which does not collapse to a 1-complex. It is composed of two Bing's houses, $\mathcal{B}_{2}$ and $\mathcal{B}_{2}^{\prime}$, each with one thick wall triangulated as in Example 2 and whose central edges $\{8,9\}$ and $\left\{8^{\prime}, 9^{\prime}\right\}$ have been identified (we use $0^{\prime}, 1^{\prime}, \ldots, 9^{\prime}$ for labeling the vertices of the thick wall of $\mathcal{B}_{2}^{\prime}$ accordingly to Figure 2(a)). In more intuitive words, we "paste" the two central edges together, by identifying vertices 8 and 9 with 8 ' and $9^{\prime}$, respectively.

In order to collapse $\mathcal{B}_{3}$ to a 1-complex, we should be able to start removing the $2 D$ bottom part of either $\mathcal{B}_{2}$ or $\mathcal{B}_{2}^{\prime}$, and for this either the edge $\{0,1\}$ or $\left\{0^{\prime}, 1^{\prime}\right\}$ must become a free face after collapsing the corresponding thick wall $\mathcal{W}$ or $\mathcal{W}^{\prime}$. Let us assume, without lose of generality, that $\{0,1\}$ becomes a free face. According to Remark 1, W must collapse from faces which does not lie in rectangles $(0,3,5,6)$ and $(1,2,4,7)$. However, the edge $\{8,9\}=\left\{8^{\prime}, 9^{\prime}\right\}$ should become a free face at some step, and this is only possible if $\mathcal{W}^{\prime}$ collapses from its faces on either the rectangle $\left(0^{\prime}, 3^{\prime}, 5^{\prime}, 6^{\prime}\right)$ or $\left(1^{\prime}, 2^{\prime}, 4^{\prime}, 7^{\prime}\right)$; that is, if $\mathcal{B}_{2}^{\prime}$ collapses 
on a 2-complex consisting of a Bing's house with two thin walls and the 1-cell $\left\{8^{\prime}, 9^{\prime}\right\}$, in which the edge $\left\{0^{\prime}, 1^{\prime}\right\}$ is not a free face. This enables us to remove all the cells in $\mathcal{B}_{2}$, after which there are not more free faces. Hence, $\mathcal{B}_{3}$ cannot collapse to a 1-complex, however it collapses on a Bing's house with two thin walls instead.

\section{The 2D Case}

First we recall an $N P$-completeness result concerning simplicial 2-complexes from EG96. Given a simplicial 2-complex $\mathcal{C}$, a $2-$ cell of $\mathcal{C}$ is called internal if it has no free faces. We denote by $\operatorname{er}(\mathcal{C})$ the minimum number of internal 2 -cells which need to be removed from $\mathcal{C}$ so that the resulting complex collapses to a 1 -complex. For instance, $\mathcal{C}$ collapses to a $1-$ complex iff $\operatorname{er}(\mathcal{C})=0$, and for a $2 \mathrm{D}$ hollow cube (or sphere) $\mathcal{C}$, we have $\operatorname{er}(\mathcal{C})=1$.

\section{GENERALIZED 2D 1-COLLAPSING:}

INSTANCE: a finite simplicial 2-complex $\mathrm{C}$ and a non-negative integer $\mathrm{k}$ QUESTION: is er (C) equal to $\mathrm{k}$ ?

Theorem 1 ([EG96]). GENERALIZED 2D 1-COLLAPSING is NP-complete.

Now, let us consider the following problem for a fixed $k \in \mathbb{N}$ :

k-GENERALIZED 2D 1-COLLAPSING:

INSTANCE: a finite simplicial 2-complex C

QUESTION: is er $(C)$ equal to $\mathrm{k}$ ?

Theorem 2. For any fixed $k$, the problem $k$-GENERALIZED 2D 1-COLLAPSING is polynomial.

Lemma 1. Let $\mathcal{C}$ be a simplicial 2-complex which is collapsible to a 1-complex and let $c$ be a 2 -cell of $\mathcal{C}$ with a free face $f$. Then the complex $\mathcal{C}_{1}$ obtained by elementary collapsing $c$ from $f$ also collapses to a 1-complex.

Lemma 1 follows from the fact that the 2 -cells of $\mathcal{C}_{1}$ can be collapsed in the same order as they are collapsed when reducing $\mathcal{C}$ to a $1-$ complex, since the removal of $c$ and $f$ does not affect the free character of $1-$ cells. Note that the 1 -complexes resulting from collapsing of $\mathcal{C}$ and $\mathcal{C}_{1}$ might be different.

Proof of Theorem 2, First we observe that the case $k=0$ follows from the polynomial character of the algorithm consisting in searching an arbitrary 2-cell with a free face, collapsing this cell, and recursively treating the resulting complex $\mathcal{C}_{1}$. From Lemma 1] either this algorithm constructs a collapsing sequence of $\mathcal{C}$ to a 1 -complex, or $\mathcal{C}$ cannot be collapsed to a 1 -complex.

Now, for the case $k \neq 0$, since $k$ is fixed, we can try all the possibilities of removal of $k 2$-cells, and try to collapse the resulting complex using the case $k=0$. The resulting algorithm, for a fixed $k$, is polynomial (in spite of an exponent $k$ in the complexity). 
Now consider the following problem:

2D POINT COLLAPSING:

INSTANCE: a finite simplicial 2-complex C

QUESTION: does C collapse to a point ?

\section{Corollary 1. The 2D POINT COLLAPSING problem is polynomial.}

Proof. We have a constructive algorithm to decide whether or not an input simplicial 2 -complex collapses to a 1 -complex. If $\mathcal{C}$ does not collapse to a 1 -complex, then, a forciori, it does not collapse to a point. Otherwise, we can construct a 1 -complex $K$ (i.e. a graph) such that $\mathcal{C}$ collapses to $K$. Then, if $K$ is not a tree, then $\mathcal{C}$ is not simply connected and does not collapse to a point. If $K$ is a tree, then $K$ (and therefore $\mathcal{C}$ ) collapses to a point. This gives a polynomial procedure to solve 2D POINT COLLAPSING.

This proof shows, in particular, that the order in which we collapse the cells of a given collapsible $2-$ complex is not important to reduce it to a single point. In the $3 \mathrm{D}$ case, however, we could get blocked on a non-collapsible complex if we choose a wrong free face at some step of a possible collapsing sequence. For instance, let $\mathcal{C}$ be a triangulated solid cube such that a Bing's house with two thin walls $\mathcal{B}$ (see Example 1) is a subcomplex of $\mathcal{C}$. Despite $\mathcal{C}$ is collapsible, it can be reduced to the non-collapsible $2-$ complex $\mathcal{B}$ by collapsing the 3 -cells which are filling both rooms from the free faces on the tunnels (see Remark 1 for another example). Anyway, we may wonder whether Theorem 2 can be generalized to the $3 \mathrm{D}$ complexes case. Section 3 shows that this is impossible (unless $P=N P$ ), since the problem of deciding whether a simplicial 3 -complex collapses to a 1 -complex is proved to be $N P$-complete.

\section{The 3D Collapsing Problem}

In the sequel, we shall consider the following problem:

3D 1-COLLAPSING:

INSTANCE: a finite simplicial 3-complex C

QUESTION: does C collapse to a 1-complex ?

Concerning the encoding of the instance, which is a simplicial 3-complex $\mathcal{C}$, we suggest that the number of vertices can be written in binary, followed by, for each simplex $s$ of $\mathcal{C}$, the binary expansions of all the numbers of vertices of $s$. Note that we could have written the number of vertices in unary and listed only the maximal simplices (i.e. those which are proper face of no simplices), but, for $3 \mathrm{D}$ complexes, this makes no significant difference concerning the size of the input.

Now we can state our main result:

Theorem 3. The 3D 1 - COLLAPSING problem is NP-complete. 
First note that 3D 1 - COLLAPSING is easily seen to be in NP since, given a simplicial 3 -complex $\mathcal{C}=(V, S)$, we can guess a sequence $\sigma=\left(\left(s_{1}, f_{1}\right), \ldots,\left(s_{p}, f_{p}\right)\right)$ of couples of cells, and check in polynomial time that $\sigma$ is a collapsing sequence to a 1-complex, that is, the cell $f_{i}$ is a maximal proper face of $s_{i}$, for $i=1, \ldots, p$, and $f_{i}$ is a free face in the complex obtained by removing the cells $s_{1}, f_{1}, \ldots, s_{i-1}, f_{i-1}$, and the cells which are not of the form $s_{i}$ or $f_{i}$ have dimension less than or equal to 1 .

The proof of Theorem 3 therefore reduces to proving that 3D 1 - COLLAPSING is NP-hard. This is made by polynomially reducing the $3-\mathrm{SAT}$ problem to 3D 1 - COLLAPSING. So, in the sequel of this section, $U$ is a finite set of $n$ boolean variables, and $C=\left\{c_{1}, \ldots, c_{m}\right\}$ is a finite collection of $m 3$-clauses. These represent an instance $I$ of the 3 - SAT problem, and we are going to construct an instance of a simplicial 3 -complex $\mathcal{C}(I)$ which is collapsible to a 1 -complex iff $I$ is satisfiable.

The complex $\mathcal{C}(I)$ is made up of several other complexes, representing literals, the clauses and the conjunction of clauses of $I$, which are connected by identifying some of their edges. The main idea is to represent the literals $u$ and $\bar{u}$, for each variable $u \in U$, by two complexes $\mathcal{C}(u)$ and $\mathcal{C}(\bar{u})$, respectively, which are related in such a way that only one of them may collapse on a 1 -complex at a first stage of a possible collapsing sequence of $\mathcal{C}(I)$. If the complex $\mathcal{C}(l)$, representing the literal $l$, could not be reduced to a 1 -complex at this stage, we will obtain the necessary feedback to collapse it through a particular edge $f(l)$, which is identified with an edge in the complex $\mathcal{C}_{\text {and }}$ representing the conjunction of clauses. In a second stage, each complex $\mathcal{C}(c)$ representing a clause $c=l_{1} \vee l_{2} \vee l_{3}$ will be collapsible to a 1 -complex if and only if at least one of the complexes $\mathcal{C}\left(l_{j}\right), j \in\{1,2,3\}$, has collapsed in the previous stage. Then $\mathcal{C}_{\text {and }}$, and hence $\mathcal{C}(I)$ through the edges $f(l)$, turns out to be collapsible if all the complexes $\mathcal{C}\left(c_{i}\right)$, $i \in\{1, \ldots, m\}$, have been collapsed. We next describe the complexes involved in this construction.

The complex $\mathcal{C}_{\text {and }}$ is just a Bing's house with one thick wall whose $2 \mathrm{D}$ walls have been triangulated in such a way that, for each literal $l$, we get an edge $f(l)$ lying at the intersection between two outer walls of the lower room (see Figure 3(a). As it was shown in Remark 1, the only possibility to reduce $\mathcal{C}_{\text {and }}$ to a 1 -complex is to collapse the thick wall until the edge $e_{\text {and }}=\{0,1\}$ becomes a free face. For this reason, we paste to this edge a simplicial representation $\mathcal{C}\left(c_{i}\right)$ of each clause $c_{i}, i \in\{1, \ldots, m\}$. This way, $\mathcal{C}_{\text {and }}$ intuitively represents the "conjunction" of the complexes $\mathcal{C}\left(c_{i}\right)$, since it will be collapsible only if each $\mathcal{C}\left(c_{i}\right)$ is previously reduced to a 1 -complex.

In order to describe the complexes $\mathcal{C}\left(c_{i}\right)$ we may assume that a boolean variable $u$ appears at most (positively or negatively) once in a given clause. If $c_{i}$ contains only one literal, then $\mathcal{C}\left(c_{i}\right)$ consists just of a single edge, specifically the edge $e_{\text {and }}$ of $\mathcal{C}_{\text {and }}$, which is also labeled with this literal. If the clause $c_{i}$ contains two literals, then it is simply represented by a triangle, one edge of this triangle being the edge $e_{\text {and }}$ of $\mathcal{C}_{\text {and }}$, and the two other edges being labeled each by one literal appearing in the clause $c_{i}$. Finally, if $c_{i}$ contains three literals (see 


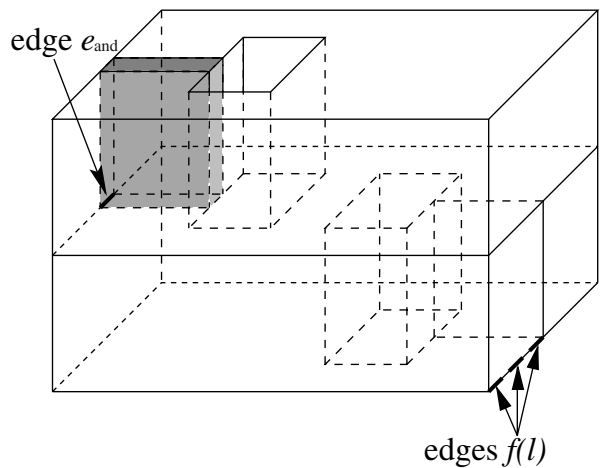

(a) The conjunction Bing's house $\mathcal{C}_{\text {and }}$.

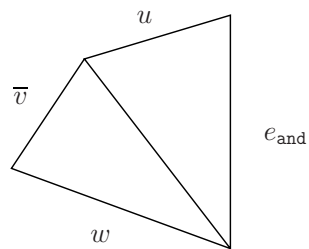

(b) Representation of a clause $c_{j}$.

Fig. 3. How to represent 3-clauses

Figure 3(b) for an example with $\left.c_{i}=u \vee \bar{v} \vee w\right)$, the clause is represented by two adjacent triangles, being $e_{\text {and }}$ one the four edges which are not shared by the two triangles and the three other of these edges being labeled each by one of the three literals of the clause $c_{i}$. By identifying all these edges, the union of the complexes $\mathcal{C}_{\text {and }}, \mathcal{C}\left(c_{1}\right), \ldots, \mathcal{C}\left(c_{m}\right)$ yields a simplicial complex $\mathcal{C}$ in which some edges are labeled by literals, and $\mathcal{C}$ can be collapsed to a 1 -complex if for each clause we use, as a free face, an edge labeled by a literal of the clause.

Now we have to paste to $\mathcal{C}$ some representation of literals. This is the most tricky point. For this purpose we shall consider, for each variable $u \in U$, two Bing's houses with two thick walls such as the one represented in Figure 4 , one, denoted by $\mathcal{C}(u)$, for the literal $u$, and one, denoted by $\mathcal{C}(\bar{u})$, for the literal $\bar{u}$. More precisely, for a given literal $l$, the two thick $3 \mathrm{D}$ walls of the complex $\mathcal{C}(l)$, denoted by $\mathcal{W}(l)$ and $\mathcal{F}(l)$ respectively, are triangulated as the thick wall in Example 2, while the triangulation of its 2D walls provides us with $m$ edges $e_{1}(l), \ldots, e_{m}(l)$ (we remind the reader that $m$ is the number of clauses), these edges lying on an outer wall (or at the intersection between two outer walls as represented in Figure 4 of the lower room of $\mathcal{C}(l)$. Then, each Bing's house $\mathcal{C}(l)$ associated to a literal $l$ is double linked to the complex $\mathcal{C}$. Firstly, for each clause $c_{j}, j \in\{1, \ldots, m\}$, in which the literal $l$ appears, we identify the edge $e_{j}(l)$ with the edge labeled by $l$ in the complex $\mathcal{C}\left(c_{j}\right)$ representing the clause $c_{j}$. Secondly, the edge $f(l)$ of the thick wall $\mathcal{F}(l)$ of $\mathcal{C}(l)$ is also identified with the edge of the same label in the complex $\mathcal{C}_{\text {and }}$ representing the conjunction of clauses (see Figures 3(a) and 4 ). Finally, for each variable $u \in U$, the literal $u$ must somehow be related to the literal $\bar{u}$ by an exclusion principle. For this, we simply identify the central edges of the thick walls $\mathcal{W}(u)$ and $\mathcal{W}(\bar{u})$ as in Example 3

Remark 2. As it was mentioned above, in order to collapse the complex $\mathcal{C}$, and thus $\mathcal{C}(I)$, to a 1-complex, we need for each clause $c_{j}$ at least one free edge labeled by a literal $l$ appearing in the clause. Since such an edge is identified with 


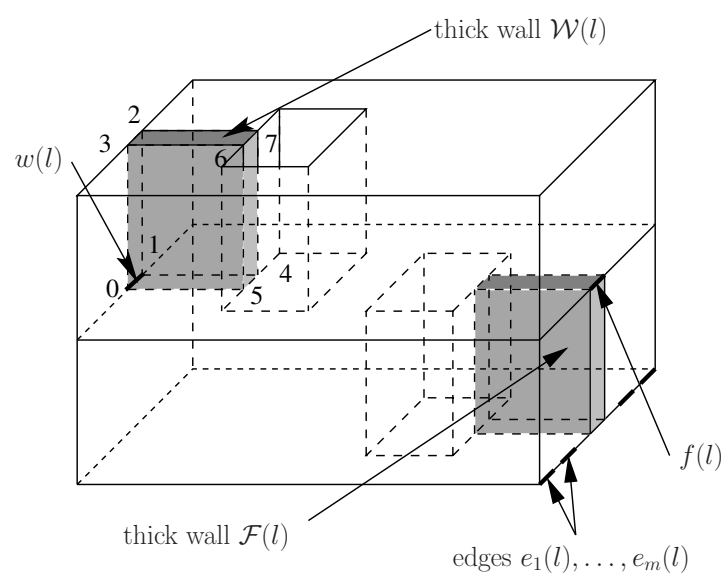

Fig. 4. The Bing's house $\mathcal{C}(l)$ with two thick walls associated to a literal $l$

the edge $e_{j}(l)$ lying in the bottom room of the Bing's house $\mathcal{C}(l)$, we must first collapse these complexes. For this, we can proceed as in Example 2 to collapse the thick wall $\mathcal{F}(l)$ of $\mathcal{C}(l)$. But in the complex obtained from $\mathcal{C}(I)$ after this process, there are three 2 -cells having the edge $f(l)$ as a face (two of them in $\mathcal{C}_{\text {and }}$ and the third one in $\mathcal{C}(l))$. So, $f(l)$ is not a free face and it cannot be used to remove the remaining cells of $\mathcal{C}(l)$. Thus, we must follow a different collapsing sequence. Indeed, we must collapse the thick walls $\mathcal{W}(l)$ and $\mathcal{W}(\bar{l})$ simultaneously as in Example 3 in order the edge $w(l)$ of $\mathcal{W}(l)$ becomes a free face of the resulting complex (see Figure 4), from which we can remove the 2-cells of (the bottom room of) $\mathcal{C}(l)$. Notice that, after this process (in which the thick walls $\mathcal{W}(l)$ and $\mathcal{W}(\bar{l})$ play the role of $\mathcal{W}$ and $\mathcal{W}^{\prime}$ in Example [3, respectively), the edge $w(\bar{l})$ of $\mathcal{W}(\bar{l})$ is not free in the resulting complex; so that, we must wait until the edge $f(\bar{l})$ becomes free (i.e., until $\mathcal{C}_{\text {and }}$ has been collapsed) to reduce $\mathcal{C}(\bar{l})$ to a 1 -complex.

The following proposition clearly follows from the construction of $\mathcal{C}(I)$.

Proposition 1. Given the data of the instance $I$ of the $3-\mathrm{SAT}$, we can construct the complex $\mathcal{C}(I)$ in polynomial time.

Then the NP-completeness of the 3D 1 -COLLAPSING problem immediately follows from the following

Theorem 4. The $3 D$ simplicial complex $\mathcal{C}(I)$ collapses to a 1 -complex if and only if the instance $I$ of the 3 - SAT problem is satisfiable.

Proof. First, assume that the instance $I$ is satisfiable, and let $G: U \longrightarrow\{0,1\}$ be a truth assignment which satisfies the collection $C$ of clauses of $I$. Let us show how to collapse $\mathcal{C}(I)$ to a 1 -complex.

We extend the truth assignment $G$ to literals by setting $G(\bar{u})=\neg G(u)$ for $u \in U$. For each literal $l$ such that $G(l)=1$, we collapse the Bing's house $\mathcal{C}(l)$, as 
it suggested in Remark 2 so that the edges labeled by $l$ in the complex $\mathcal{C}$ become free. Thus, since $G$ is a satisfying truth assignment, for each clause $c_{j} \in C$ there is a free edge $e_{j}(l)$ in the resulting complex from which we can remove the (at most) two triangles of $\mathcal{C}\left(c_{j}\right)$. Therefore, we can collapse the thick wall of the Bing's house $\mathcal{C}_{\text {and }}$ as in Example 2 until the edge $e_{\text {and }}$ becomes free, which allows us to remove the remaining 2 -cells of $\mathcal{C}_{\text {and }}$. Finally, since the faces $f(l)$, for all literals $l$ (in particular for those with $G(l)=0$ ), are faces of no 2 -cells of the remaining of $\mathcal{C}_{\text {and }}$, we can begin to collapse the thick wall $\mathcal{F}(l)$ similarly to Example 2, and then collapse all Bing's houses $\mathcal{C}(l)$ to a 1 -complex.

Conversely, suppose that $\mathcal{C}$ collapses to a 1 -complex $K$. For $l$ literal of $I$, let $G(l)$ be equal to 1 if there exists and edge $e_{j}(l)$ in $\mathcal{C}(l)$ which is removed before the edge $f(l)$ (in particular if $f(l)$ is an edge of $K$ ) and equal to 0 otherwise. Let us prove that for any literal $l$ we have $G(l)=1$ implies $G(\bar{l})=0$, and that the truth assignment $G^{\prime}$ defined by $G^{\prime}(l)=G(l)$ if $G(l)=1$ or $G(\bar{l})=1$ and by $G^{\prime}(u)=1$ and $G^{\prime}(\bar{u})=0$ for all $u \in U$ such that $G(u)=G(\bar{u})=0$ is a satisfying truth assignment for $I$.

First, assume that $G(l)=1$. We need collapse (at least part of) the bottom room of $\mathcal{C}(l)$ in order to remove the edge $e_{j}(l)$. For this, according to Remark 1 we must start collapsing from either the edge $w(l)$ or $f(l)$ of $\mathcal{C}(l)$. Thus, since $e_{j}(l)$ is removed before $f(l)$, this process starts being the edge $w(l)$ a free face, for which necessarily the thick walls $\mathcal{W}(l)$ and $\mathcal{W}(\bar{l})$ must be collapsed simultaneously as in Example 3. As a consequence the edge $w(\bar{l})$ is not free in the remaining of $\mathcal{C}(\bar{l})$, and hence no edge $e_{j}(\bar{l})$ can be removed before $f(\bar{l})$. This shows that $G(\bar{l})=0$.

Now let us prove that $G^{\prime}$ is a satisfying truth assignment for $I$. In order to remove the 2 - cells of the Bing's house $\mathcal{C}_{\text {and }}$ by collapsing, we have to use the edge $e_{\text {and }}$ as a free face by Remark 1. Therefore, for each clause $c_{j}, j \in\{1, \ldots, m\}$, there is an edge labeled by some literal of the clause in the (at most) two triangles representing the clause $c_{j}$ (see Figure $3(\mathrm{~b})$ which is used as a free face before any edge of the form $f(l)$ is removed. This shows that in each clause $c_{j}$ appears a literal $l$ such that $G(l)=1$, so that $G^{\prime}$ is a satisfying truth assignment for $I$.

\section{Conclusion}

We proved that deciding whether a simplicial 3-complex collapses to a 1complex is an intractable problem. This is, as far as we know, the first result of that kind concerning topology preservation. Several kindred questions remain:

- Characterizing the complexity of the problem of deciding whether a simplicial 3-complex collapses to a point;

- Studying the complexity of characterization of simple points in high dimensional digital spaces ([K97, [KR01, [B99]);

- Characterizing the complexity of the problem of deciding whether a 3D digital object can be reduced to another object by sequential deletion of simple points $([\mathrm{F} 00])$. 


\section{References}

[B99] Bertrand, G.: New Notions for Discrete Topology. In: Bertrand, G., Couprie, M., Perroton, L. (eds.) DGCI 1999. LNCS, vol. 1568, Springer, Heidelberg (1999)

[B64] Bing, R.H.: Some aspects of the topology of 3-manifolds related to the Poincaré conjecture. In: Saaty, T.L. (ed.) Lectures on Modern Mathematics, vol. II, pp. 93-128. Wiley, Chichester (1964)

[C71] Cook, S.A.: The complexity of Theorem Proving Procedures. In: Proc. 3rd Ann. ACM Symp. on Theory of Computing, Association for Computing Machinery, New-York, pp. 151-158

[EG96] Egecioglu, O., Gonzalez, T.F.: A Computationally Intractable Problem on Simplicial Complexes. Computational Geometry, Theory and Applications 6, 8598 (1996)

[F00] Fourey, S., Malgouyres, R.: A concise characterization of 3D simple points. Discrete Applied Mathematics 125(1), 59-80 (2003)

[GJ79] Garey, M.R., Johnson, D.S.: Computers and Intractability: a guide to the theory of $N P$-completeness. W.H. Freeman and Company publishers, New York

[K97] Kong, T.Y.: Topology-Preserving Deletion of 1's from 2-, 3- and 4- Dimensional Binary Images. In: Ahronovitz, E. (ed.) DGCI 1997. LNCS, vol. 1347, pp. 3-18. Springer, Heidelberg (1997)

[KR01] Kong, T.Y., Roscoe, A.W.: Simple Points in 4-dimensional (and HigherDimensional) Binary Images (paper in preparation)

[VL90] : Handbook of theoretical computer science. In: Van Leeuwen, J. (ed.) Algorithms and complexity, vol. A, pp. 67-161. Elsevier Science Publishers, Amsterdam (1990) 